\title{
Efeitos da fisioterapia aquática no equilíbrio em pacientes com doença em Parkinson
}

\author{
Effects of aquatic physiotherapy on balance in patients with Parkinson's disease \\ Efectos de la fisioterapia acuática en el equilibrio en pacientes con enfermedad de Parkinson
}

\author{
José Edmilson da Silva Neto \\ ORCID: https://orcid.org/0000-0002-6372-0481 \\ Faculdade de Ensino Superior do Piaú, Brasil \\ E-mail: edmilson161214@hotmail.com \\ Ester Miranda de Sousa \\ ORCID: https://orcid.org/0000-0003-2319-4840 \\ Faculdade de Ensino Superior do Piauí, Brasil \\ E-mail: mirandaester70@gmail.com \\ Luigi Gabriel Brasil da Silva \\ ORCID: https://orcid.org/0000-0002-8556-3958 \\ Faculdade de Ensino Superior do Piauí, Brasil \\ E-mail: gabrie1010@outlook.com \\ Diane Nogueira Paranhos Amorim \\ ORCID: https://orcid.org/0000-0002-5362-5747 \\ Faculdade de Ensino Superior do Piauí, Brasil \\ E-mail: dnpa.fisio@gmail.com \\ Ruth Raquel Soares de Farias \\ ORCID: https://orcid.org/0000-0002-0988-0900 \\ Faculdade de Ensino Superior do Piauí, Brasil \\ E-mail: ruthraquelsf@gmail.com \\ Francisco Maurilio da Silva Carrías \\ ORCID:https://orcid.org/0000-0002-0762-0494 \\ Faculdade de Ensino Superior do Piauí, Brasil \\ E-mail: mauriliocarrias@gmail.com \\ Thais Cristina da Costa Rocha Pereira \\ ORCID: https://orcid.org/0000-0002-2020-6722 \\ Faculdade de Ensino Superior do Piauí, Brasil \\ E-mail:thaisinha.ibr@gmail.com
}

\begin{abstract}
Resumo
Introdução: A doença de Parkinson (DP) é uma patologia lenta e crônica do sistema nervoso, na qual ocorre uma degeneração nas células dos gânglios basais ocasionando uma perda ou interferência na ação da dopamina. O Objetivo desse estudo foi verificar a eficácia da Fisioterapia aquática (FA) no equilíbrio em pacientes com doença de Parkinson por meio de uma revisão sistemática de literatura. Métodos: É uma revisão sistemática da literatura, que utilizou-se as bases de dados eletrônicas Literatura Latino-Americana e do Caribe em Ciências da Saúde (LILACS), MEDlars on LINE (MEDLINE), National Library of Medicine and National Institute of Health (PUBMED), Scientific Electronic Library Online (SciELO) e Physiotherapy Evidence Database (PEDro) e teve como estratégia de busca o cruzamentos desse descritores com os operadores booleanos AND e OR. Resultados e Discussão: Os resultados mostraram que a FA tem um papel significativo na manutenção do equilíbrio. Exercícios realizados em terra com a FA na restauração do equilíbrio em pacientes com Doença de Parkinson, mostrou que exercícios realizados no meio aquático foram mais eficazes e viáveis para esse tipo de paciente. Considerações finais: A FA é uma boa alternativa para otimização do equilíbrio, da instabilidade postural e, consequentemente, para a redução do número de quedas em pacientes idosos com DP, repercutindo positivamente na $\mathrm{QV}$ desses pacientes.
\end{abstract}

Palavras-chave: Hidroterapia; Doença de Parkinson; Equilíbrio Postural.

\begin{abstract}
Introduction: Parkinson's disease (PD) is a slow and chronic pathology of the nervous system, in which there is a degeneration in the cells of the basal ganglia, causing a loss or interference in the action of dopamine. The aim of this study was to verify the effectiveness of Aquatic Physical Therapy (AF) in balance in patients with Parkinson's disease through a systematic literature review. Methods: This is a systematic review of the literature, using the electronic databases of Latin American and Caribbean Literature in Health Sciences (LILACS), MEDlars online (MEDLINE), National Library of Medicine and National Institute of Health (PUBMED). ), Scientific Electronic Library Online (SciELO) and Physiotherapy Evidence Database (PEDro) and had as a search strategy the crossing of these descriptors with the Boolean operators AND and OR. Results and Discussion: The results showed that AF plays a significant role in maintaining balance. Exercises performed on land with the AF to restore balance in patients with
\end{abstract}


Parkinson's Disease, showed that exercises performed in the aquatic environment were more effective and viable for this type of patient. Final considerations: The AF is a good alternative for optimizing balance, of postural instability and, consequently, to the reduction in the number of falls in elderly patients with PD, with positive repercussions on the QoL of these patients.

Keywords: Hydrotherapy; Parkinson's disease; Postural Balance.

\section{Resumen}

Parkinson's disease (PE) is a slow and chronic pathology of the nervous system, in which a degeneration of the cells of the basal ganglia takes place, causing a loss or interference in the action of dopamine. The objective of this study was to verify the effectiveness of the Physical Aquatic Therapy (AF) in the balance in patients with Parkinson's disease through a systematic review of the literature. It is a systematic literature review using electronic databases Latin American and Caribbean Literature in Health Sciences (LILACS), MEDlars online (MEDLINE), National Library of Medicine and National Institute of Health (PUBMED), Scientific Electronic Library Online (SciELO ) and Physiotherapy Evidence Database (PEDro) and as a search strategy to cross these descriptors with the Boolean operators AND and OR. The results showed that FA plays an important role in the maintenance of balance. The exercises carried out on land with the AF to restore the balance in patients with Parkinson's disease, showed that the exercises carried out in the aquatic environment were more effective and viable for this type of patient. FA is a good alternative to optimize balance, postural instability and, consequently, to reduce the number of falls in elderly patients with PE, with positive repercussions on the CV of these patients.

Palabras clave: Hidroterapia; Enfermedad de Parkinson; Equilibrio Postural.

\section{Introdução}

A doença de Parkinson (DP) é uma patologia lenta e crônica do sistema nervoso, onde ocorre uma degeneração nas células dos gânglios basais ocasionando uma perda ou interferência na ação da dopamina, que é o principal neurotransmissor dos gânglios basais, e eles contribuem para a precisão e a uniformidade dos movimentos e coordenam as mudanças de posição (Haase et al., 2008).

Os principais sintomas da Doença de Parkinson são: tremor em repouso, rigidez, bradicinesia e instabilidade postural (Filippin et al., 2014). Associados a essas alterações, o comprometimento físico-mental, emocional, social e econômico decorrente e relacionados aos sinais e sintomas da DP interferem no nível de capacidade do indivíduo e podem influenciar negativamente a qualidade de vida $(\mathrm{QV})$ do mesmo, levando-o ao isolamento e à diminuição da sua participação na vida social (Lana et al., 2007).

Para minimizar os problemas motores, como equilíbrio, de forma a manter a independência na realização das atividades da vida diária e melhorar a QV, a Fisioterapia é uma das principais intervenções não-farmacológicas. A fisioterapia também atua no aumento da mobilidade podendo modificar a progressão da doença e impedir contraturas e outras complicações secundárias (Sant et al., 2008).

Dentre as diversas intervenções fisioterapêuticas, a fisioterapia aquática (FA) vem sendo reconhecida como importante instrumento para otimização do equilíbrio dos pacientes com doença de Parkinson, pois melhora a instabilidade postural. (Palácio et al., 2011, Andrade, Silva \& Corso, 2010). A disfunção do equilíbrio (DB) é um sinal frequente e incapacitante da doença de Parkinson (DP), levando a quedas inesperadas e, portanto, relacionadas a uma pior QV (Smania et al., 2010).

Portanto, a manutenção do equilíbrio pode ter importantes implicações para a QV dos idosos com DP, por estar relacionada com a melhora QV e a capacidade de ocupar-se com o trabalho até idades mais avançadas e com atividades que lhe sejam agradáveis, e as quedas e a estabilidade postural são uma das principais causas de incapacidade em idosos com essa afecção (Barbosa, 2016).

Os exercícios aquáticos têm sido amplamente usados em programas de fisioterapia para diferentes doenças (Silva et al., 2019).

A flutuabilidade da água reduz a gravidade e um o ambiente aquático pode ser considerado um ambiente de microgravidade.

Muitos autores documentaram modificações de estática e dinâmica controle postural em microgravidade prolongada. Modificações do controle do corpo, devido a disfunções do vestibular informações que ocorrem debaixo de água, sublinhe o 
papel principal do sistema proprioceptivo para postural controle neste ambiente. Ambiente aquático permite o treino de equilíbrio em condições seguras, evitando quedas e reduzindo o medo de cair (Baroni et al., 2001).

Perante o que foi apresentado, tem-se o seguinte questionamento: Qual a contribuição da FA no equilíbrio dos idosos com doenças de parkinson? Sendo assim o objetivo desse trabalho foi analisar o efeito da FA no equilíbrio em pacientes com doença de Parkinson por meio de uma revisão sistemática de literatura.

\section{Metodologia}

A revisão sistemática da literatura é uma metodologia rigorosa proposta para: identificar os estudos sobre um tema em questão, aplicando métodos explícitos e sistematizados de busca (De-La-Torre-Ugarte-Guanilo,Takahashi\& Bertolozzi, 2011). A revisão sistemática da literatura tem como objetivo responder a uma pergunta específica, que deve ser realizada de acordo com um projeto pré-estabelecido e ter uma hipótese para demonstrar ou rejeitar (Araújo \& Alonso, 2011).

Este estudo trata-se de uma revisão Sistemática da literatura, que reuniu amostras de estudos científicos referentes ao efeito da FA no equilíbrio de pacientes idosos com doença de Parkinson, tendo como pergunta norteadora: "Qual o efeito da fisioterapia aquática no equilíbrio de pacientes com doenças de Parkinson?”

A pesquisa foi realizada nas bases de dados eletrônicas Literatura Latino-Americana e do Caribe em Ciências da Saúde (LILACS), MEDlars onLINE (MEDLINE), National Library of Medicine and National Institute of Health (PUBMED), Scientific Electronic Library Online (SciELO) e Physiotherapy Evidence Database (PEDro) por meio da consulta pelas subsequentes Descritores: hidroterapia, equilíbrio, idosos e Parkinson durante os meses de agosto e novembro de 2021, e teve como estratégia de busca o cruzamentos desse descritores com os operadores booleanos AND e OR.

Adotou-se como critérios de inclusão: artigos originais do tipo experimentais, estudos de caso, estudos observacionais publicados entre os anos 2013 a 2021 nos idiomas português, inglês e espanhol que tinham como amostra Pacientes diagnosticados com doença de Parkinson. Os critérios de exclusão dos artigos foram: Tese, monografias, dissertações e artigos que fogem do tema abordado.

A busca dos artigos foi realizada através de dois revisores independentes. A seleção dos artigos foi feita inicialmente pelo título e resumo, logo após, os artigos foram lidos na íntegra e selecionados seguindo os critérios de inclusão e exclusão.

A metodologia dos artigos selecionados foi revisada e avaliada pela escala PEDro, utilizada na área de reabilitação, que avaliam a qualidade metodológica dos estudos, a escala tem uma pontuação total de 10 pontos, sendo que escores $\geq 5$ são considerados de alta qualidade. Os artigos selecionados foram expostos através de uma tabela de resultados, e analisados a partir da leitura na íntegra (Moseley et al., 2002).

\section{Resultados}

Dos 61 artigos encontrados, quatro foram incluídos. Dentre os artigos incluídos, três são internacionais e somente um nacional. As amostras dos estudos são pacientes diagnosticados com DP com a idade entre 45 a 86 anos. A síntese do processo de inclusão e exclusão dos artigos encontra-se no Fluxograma 1. 
Figura 1: Fluxograma do processo de inclusão e exclusão de artigos.

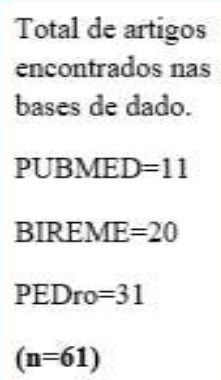

Artigos selecionados pelo título.

$(\mathrm{n}=\mathbf{2 1})$
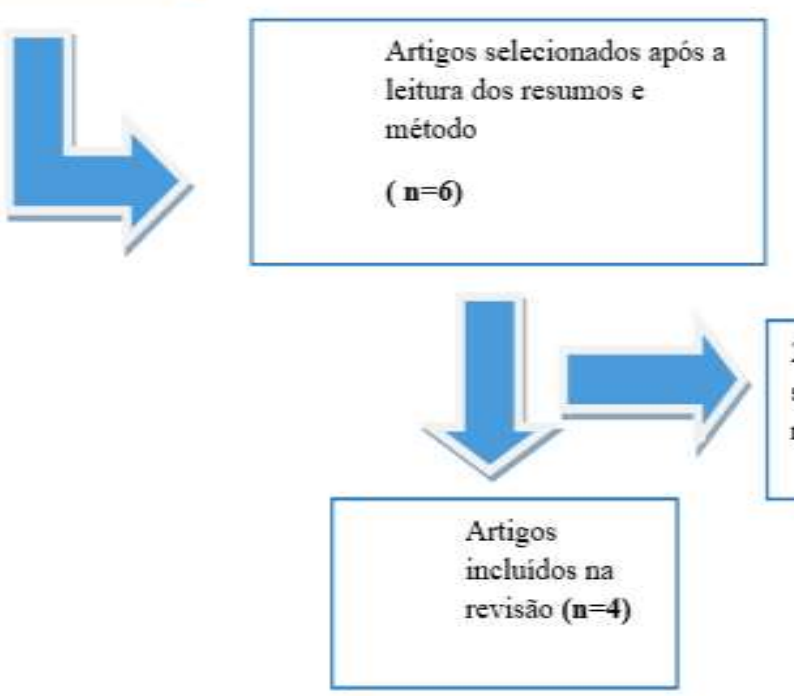

Fonte: Autores.

Os resultados dos artigos selecionados foram analisados criteriosamente e exibidos no Quadro 1. 
Quadro 1 - Demonstração dos resultados dos artigos analisados.

\begin{tabular}{|c|c|c|c|c|c|}
\hline Autor/Ano & Título & $\begin{array}{c}\text { Tipo de } \\
\text { estudos/Amostra }\end{array}$ & Objetivo & Protocolo de exercícios & Principais resultados \\
\hline $\begin{array}{l}\text { Silva et al., } \\
\text { (2013) }\end{array}$ & $\begin{array}{c}\text { Efeitos da } \\
\text { fisioterapia } \\
\text { aquática na } \\
\text { qualidade de vida } \\
\text { de sujeitos com } \\
\text { doença de } \\
\text { Parkinson }\end{array}$ & $\begin{array}{c}\text { Tipo estudo: } \\
\text { Intervenção de caráter } \\
\text { longitudinal } \\
\text { Amostra: } 13 \text { pacientes, } \\
\text { sendo } 6 \text { do sexo } \\
\text { masculino e } 7 \text { do sexo } \\
\text { feminino com DP }\end{array}$ & $\begin{array}{l}\text { Avaliar os efeitos da } \\
\text { FA na QV de } \\
\text { pacientes com DP nos } \\
\text { estágios de leve a } \\
\text { moderado utilizando- } \\
\text { se do PDQ-39 }\end{array}$ & $\begin{array}{l}\text { Consistiu de } 16 \text { sessões com duração de } 1 \\
\text { hora. } \\
\text { Aquecimento: duração de cinco minutos, } \\
\text { sendo composta por duas voltas de } \\
\text { caminhada frontal, lateral e posterior dentro } \\
\text { da piscina } \\
\text { Alongamento: alongamentos passivos, que } \\
\text { evoluíram para ativos musculaturas: MMSS } \\
\text { MMII } \\
\text { Fase 3: foram realizados exercícios ativos } \\
\text { resistidos na posição ortostática } \\
\text { Relaxamento: O relaxamento era promovido } \\
\text { através de flutuadores e massagens de } \\
\text { turbilhonamento }\end{array}$ & $\begin{array}{c}\text { Foi possível identificar } \\
\text { uma melhor percepção } \\
\text { da QV dos pacientes } \\
\text { após participar do } \\
\text { programa de } \\
\text { exercícios na FA, } \\
\text { principalmente com } \\
\text { relação aos domínios } \\
\text { de estigma, } \\
\text { desconforto físico, } \\
\text { mobilidade,equilíbrio } \\
\text { e comunicação. }\end{array}$ \\
\hline $\begin{array}{l}\text { Volpe } \text { et al., } \\
\text { (2014) }\end{array}$ & $\begin{array}{l}\text { Comparing the } \\
\text { effects of } \\
\text { hydrotherapy and } \\
\text { land-based therapy } \\
\text { on balance in } \\
\text { patients with } \\
\text { Parkinson's } \\
\text { disease: a } \\
\text { randomized } \\
\text { controlled pilot } \\
\text { study }\end{array}$ & $\begin{array}{c}\text { Tipo de estudo:Um } \\
\text { ensaio randomizado } \\
\text { simples-cego } \\
\text { controlado. } \\
\text { Amostra:34 pacientes } \\
\text { elegíveis, } \\
\text { diagnosticados com } \\
\text { DP. }\end{array}$ & $\begin{array}{l}\text { Avaliar a viabilidade } \\
\text { de um tratamento de } \\
\text { FA em Pacientes com } \\
\text { doença de Parkinson } \\
\text { com estágio moderado } \\
\text { de doença e comparar } \\
\text { a eficácia deste } \\
\text { tratamento em } \\
\text { parâmetros de } \\
\text { equilíbrio com os } \\
\text { tradicionais } \\
\text { fisioterapias terrestre }\end{array}$ & $\begin{array}{l}60 \text { minutos de tratamento, cinco dias por } \\
\text { semana durante dois meses Aquecimento: } \\
\text { Compreendeu um aquecimento } \\
\text { cardiovascular e alongamento exercícios por } \\
10 \text { minutos, seguidos por } 40 \text { minutos } \\
\text { treinamento de equilíbrio baseado em } \\
\text { perturbação e um treinamento de } 10 \text { minutos } \\
\text { esfriar. } \\
\text { Exercícios para treinamento de força e } \\
\text { equilíbrio baseado em perturbação do } \\
\text { equilíbrio, treinamento, com ênfase no } \\
\text { alcance funcional, testes em condições } \\
\text { protegidas e ativação das respostas posturais } \\
\text { às perturbações externas. }\end{array}$ & $\begin{array}{l}\text { FA pode ser um } \\
\text { possível tratamento } \\
\text { para o equilíbrio } \\
\text { disfunção em } \\
\text { pacientes } \\
\text { parkinsonianos com } \\
\text { um estágio moderado } \\
\text { da doença, com } \\
\text { potencial para } \\
\text { melhorar estabilidade } \\
\text { postural, reduzindo a } \\
\text { taxa de quedas em } \\
\text { pessoas protegidas } \\
\text { condições. }\end{array}$ \\
\hline $\begin{array}{l}\text { Palamara et } \\
\text { al. (2017) }\end{array}$ & $\begin{array}{l}\text { Land Plus Aquatic } \\
\text { Therapy Versus } \\
\text { Land-Based } \\
\text { Rehabilitation } \\
\text { Alone for the } \\
\text { Treatment of } \\
\text { Balance } \\
\text { Dysfunction in } \\
\text { Parkinson's } \\
\text { Disease: A } \\
\text { Randomized, } \\
\text { Controlled Study } \\
\text { with 6-Month } \\
\text { Follow-Up }\end{array}$ & $\begin{array}{l}\text { Tipo de estudo: } \\
\text { Ensaio clínico } \\
\text { randomizado } \\
\text { Amostra: } 17 \\
\text { pacientes afetados por } \\
\text { DP }\end{array}$ & $\begin{array}{l}\text { O objetivo do nosso } \\
\text { estudo foi avaliar se } \\
\text { uma intervenção física } \\
\text { terrestre específica } \\
\text { com as } 76 \text { inclusões } \\
\text { de terapia aquática é } \\
\text { mais eficaz do que a } \\
\text { reabilitação baseada } \\
\text { em terra sozinha no } \\
\text { BD de pacientes com } \\
\text { DP, imediatamente } \\
\text { após a terapia e em } \\
\text { uma avaliação de } \\
\text { acompanhamento de } \\
\text { seis meses. }\end{array}$ & $\begin{array}{l}\text { O programa de terapia aquática incluiu: } 142 \\
\text { sessões de exercícios aeróbicos e atividades } \\
\text { físicas para melhorar o equilíbrio, motoras, } \\
\text { coordenação e mobilidade das articulações. } \\
\text { Aquecimento: } 10 \text { minutos e consistiu em } \\
\text { exercícios de caminhada na água, em } \\
\text { diferentes direções, para reforçar o controle } \\
\text { do equilíbrio. } \\
\text { Fase 2:Os pacientes foram submetidos a } \\
\text { exercícios de mobilidade de tronco em pé e } \\
\text { sentado em um dispositivo flutuante }\end{array}$ & $\begin{array}{l}\text { A terapia aquática } \\
\text { adicionada a uma } \\
\text { reabilitação intensiva } \\
\text { em terra pode } \\
\text { contribuir para uma } \\
\text { melhora duradoura do } \\
\text { equilíbrio. }\end{array}$ \\
\hline $\begin{array}{l}\text { Terrens;Soh, } \\
\text { Morgan } \\
(2020)\end{array}$ & $\begin{array}{l}\text { The safety and } \\
\text { feasibility of a } \\
\text { Halliwick style of } \\
\text { aquatic } \\
\text { physiotherapy for } \\
\text { falls and balance } \\
\text { dysfunction in } \\
\text { people with } \\
\text { Parkinson's } \\
\text { Disease: A single } \\
\text { blind pilot trial }\end{array}$ & $\begin{array}{c}\text { Tipo de estudo: } \\
\text { Estudo piloto cego } \\
\text { único } \\
\text { Amostra: } 30 \\
\text { participantes }\end{array}$ & $\begin{array}{l}\text { O objetivo secundário } \\
\text { era comparar a } \\
\text { Intervenção do estilo } \\
\text { Halliwick com } \\
\text { fisioterapia tradicional } \\
\text { aquática e terrestre em } \\
\text { termos de gravidade } \\
\text { da doença, equilíbrio e } \\
\text { medo de cair }\end{array}$ & $\begin{array}{l}\text { As sessões tiveram duração de } 60 \text { minutos } \\
\text { por semana durante } 12 \text { semanas. } \\
\text { Exercícios aquáticos Halliwick (exercícios } \\
\text { básicos específicos e exercícios do conceito } \\
\text { Halliwick. } \\
\text { Exercícios de caminhada como um } \\
\text { aquecimento e tanto superior quanto } \\
\text { alongamento do membro inferior para } \\
\text { esfriar. } \\
\text { O grupo de intervenção aquática Halliwick } \\
\text { completou } \\
\text { mobilidade do tronco, estabilização central e } \\
\text { exercícios rotacionais como os exercícios } \\
\text { principais, enquanto o outros grupos de } \\
\text { intervenção completaram uma série de } \\
\text { exercícios de força, equilíbrio e aeróbicos. }\end{array}$ & $\begin{array}{c}\text { Fisioterapia aquática, } \\
\text { incluindo a abordagem } \\
\text { do conceito Halliwick, } \\
\text { é um tratamento viável } \\
\text { e seguro para pessoas } \\
\text { com DP, uma } \\
\text { população, vulnerável } \\
\text { a um alto risco de } \\
\text { quedas }\end{array}$ \\
\hline
\end{tabular}

Fonte: Autores.

\section{Discussão}

Os resultados desta revisão mostraram que a FA tem um papel significativo na manutenção do equilíbrio. O estudo realizado por Volpe et al. (2014), que comparou exercícios realizados em terra com a fisioterapia aquática na restauração do equilíbrio em pacientes com Doença de Parkinson, mostrou que exercícios realizados no meio aquático foram mais eficazes e 
viáveis para esse tipo de paciente, que apresentaram melhora quando se aplicaram a seguintes escalas: Escala de Equilíbrio de Berg, Escala de Confiança de Equilíbrio Específico de Atividades.

Terrens, et al. (2020) corroboram com esse estudo ao destacar a importância de uma modalidade aquática dentro do plano terapêutico de um paciente com DP, os autores apontam o método Halliwick como uma opção segura de tratamento aquático nessa população, pois é uma intervenção que enfoca a rotação e estabilização do tronco, e consequentemente a otimização do equilíbrio (Cavalca \& Soldi, 2004).

Esta revisão mostrou também a aceitação da terapia aquática pelos pacientes devido aos benefícios das propriedades da água sobre o corpo, que proporcionam a diminuição do impacto nas articulações e o relaxamento, promovido pela água aquecida (Palamara et al.,2017). As diferentes propriedades físicas da água (densidade, gravidade específica, pressão hidrostática, flutuação, viscosidade e termodinâmica), também podem desempenhar um papel importante na melhoria do controle de equilíbrio em pacientes com doença de Parkinson, permitindo o treinamento de equilíbrio em condições seguras, reduzindo o medo de cair e evitar e o risco de quedas (Volpe, et al 2014).

O estudo realizado por Da Silva et al. (2013), descreve a influência da FA sobre o equilíbrio visto que pode ter contribuído para a melhora da QV dos paciente com DP, pois segundo o mesmo a instabilidade postural que é um dos sinais e sintomas da doença é uma impacto na vida desses indivíduos, que está relacionada a alterações na marcha e risco de quedas.

A diminuição do risco de quedas foi citada pelos autores Volpe et al. (2014); Palamara et al. (2017) e Terrens et al. (2020), os quais enfatizaram em seus estudos, a eficácia da fisioterapia aquática na diminuição da incidência de quedas em idosos com DP, considerando que a queda é principal agente causador de lesões e fraturas nessa população.

$\mathrm{Na}$ atualidade, as quedas se tornaram um dos maiores problemas de saúde pública, devido ao aumento da morbidade, mortalidade e custos para a família e a sociedade. Os principais fatores de risco para quedas estão relacionados à história de quedas, aumento da idade, uso de medicamentos psicotrópicos, riscos ambientais, sexo feminino, déficit visual, além de limitação funcional, fraqueza muscular e déficit de equilíbrio, os quais podem ser minimizados pela FA (Antes, Schneider, D’orsi, 2015).De acordo com Franciulli (2015) a FA tem um papel essencial na reabilitação funcional desses indivíduos, visando à prevenção de quedas e a restauração de atividades de vida diária.

Como limitação do estudo, podemos apontar a escassez de artigos nacionais incluídos, deste modo, o estudo traz uma análise baseada, em grande parte, em amostras de idosos americanos. Sugere-se, portanto que mais estudos sejam realizados em diferentes amostras de idosos com DP, para maior esclarecimento dos efeitos da FA.

\section{Considerações Finais}

Este estudo, por meio da revisão de literatura, indica que a FA é uma boa alternativa para otimização do equilíbrio, da instabilidade postural e, consequentemente, para a redução do número de quedas em pacientes idosos com DP, repercutindo positivamente na QV desses pacientes. Sugerimos que mais estudos sejam realizados, de forma que possa contribuir para com para o esclarecimento da FA no equilíbrio de pacientes com DP.

\section{Referências}

Andrade, C. H. S., Silva, B. F. \& Corso, S. D (2010). Efeitos da hidroterapia no equilíbrio de indivíduos com doença de Parkinson. ConScientiae Saúde. 9(3), 317-28. http://www.redalyc.org/articulo.oa?id=92915260020.

Antes, D. L., Schneider, I. J. C. \& D’Orsi, E. (2015). Mortalidade por queda em idosos: estudo de série temporal. Revista Brasileira de Geriatria de Gerontologia. 18(4), 769-78.https://doi.org/10.1590/1809-9823.2015.14202.

Araújo Alonso., M. (2011). Las revisiones sistemáticas.Medwave, Madrid (Espanha).11. https://www.medwave.cl/link.cgi/Medwave/Series/mbe01/5220. https://10.5867/medwave.2011.11.5220. 
Barbosa., R M (2016). Educação de adultos-contribuição para a melhoria da qualidade de vida dos cuidadores informais de idosos. Tese de Doutorado. http://hdl.handle.net/10400.26/14011.

Baroni G., Pedrocchi A., Ferrigno G., Massion J \& Pedotti A. (2001). Static and dynamic postural control in longterm microgravity: evidence of a dual adaptation. J Appl Physio. 90: 205-215. https://doi.org/10.1152/jappl.2001.90.1.205.

Cavalca, C., \& Soldi, F. (2004). Avaliação da aptidão física em pacientes com doença de parkinson submetidos a tratamento hidroterápico através do método halliwick. Santa Catarina: Unisul. http://fisio-tb.unisul.br/Tccs/04b/carolina/artigocarolinacavalca.

da Silva, R. F., Vieira, A. P. O., \& Brito, A. P. (2019). Efeitos positivos da fisioterapia na depressão através do exercício físico e hidroterapia. Scire Salutis, 9(1), 1-8. https://doi.org/10.6008/CBPC2236-9600.2019.001.0001

De-la-Torre-Ugarte, M. C., Takahashi, R. F., \& Bertolozzi, M. R. (2011). Revisão sistemática: noções gerais. Revista da Escola de Enfermagem da USP, 45(5), 1260-1266. https://doi.org/10.1590/S0080-62342011000500033.

Filippin, N. T., Martins, J. S., Dela Libera, L. B., Halberstadt, B. F., \& Severo, A. R. (2014). Qualidade de vida de sujeitos com doença de Parkinson e seus cuidadores. Fisioterapia em Movimento, 27(1), 57-66. http://dx.doi.org.10.1590/0103-5150.027.001.AO06.

Franciulli, P. M. et al. (2015). Efetividade da hidroterapia e da cinesioterapia na reabilitação de idosos com histórico de quedas. Estudos Interdisciplinares sobre o Envelhecimento. 20(3). https://www.seer.ufrgs.br/RevEnvelhecer/article/view/38784.

Haase, D. C. B. V., Machado, D. C., \& de Oliveira, J. G. D. (2008). Atuação da fisioterapia no paciente com doença de Parkinson. Fisioterapia em Movimento (Physical Therapy in Movement), 21(1). https://periodicos.pucpr.br/fisio/article/view/19033.

Jankovic, J., \& Tolosa, E. (Eds.). (2007). Parkinson's disease and movement disorders. Lippincott Williams \& Wilkins.https://books.google.com.br/books?hl=pt-.

Lana R. C., Álvares, L. M. R. S., Nasciutti-Prudente, C., Goulart, F. R. P., Teixeira-Salmela, L. F., \& Cardoso, F. E. (2007). Percepção da qualidade de vida de indivíduos com Doença de Parkinson através do PDQ-39. Rev Bras Fisioter.;11(5):397-402. https://doi.org/10.1590/S1413-35552007000500011.

Moseleyam, Herbert, R. D., Sherrington, C., \& Maher, C. G. (2002). Evidence for physiotherapy practice: a survey of the physiotherapy evidence database (PEDro). Austr J Physiother. https://doi.org/10.1016/S0004-9514(14)60281-6.

Palácio, S. G., Barroca, J. B., Toldo, K. F., de Lima Ramalho, J. B., Vanzela, A. L., \& Facci, L. M. (2011). < b> Estudo Comparativo entre a Hidroterapia e a Cinesioterapia na Doença de Parkinson. Saúde e Pesquisa, 4(2). http://periodicos.unicesumar.edu.br/index.php/saudpesq/article/view/1414/1276>.

Palamara, G., Gotti, F., Maestri, R., Bera, R., Gargantini, R., Bossio, F., \& Frazzitta, G. (2017). Land plus aquatic therapy versus land-based rehabilitation alone for the treatment of balance dysfunction in Parkinson disease: a randomized controlled study with 6-month follow-up. Archives of physical medicine and rehabilitation, 98(6), 1077-1085.https://doi.org/10.1016/j.apmr.2017.01.025.

Silva, D. M. D et al. (2013). Efeitos da fisioterapia aquática na qualidade de vida de sujeitos com doença de Parkinson. Fisioterapia e Pesquisa, 20, 17-23. https://doi.org/10.1590/S1809-29502013000100004.

Smania N et al, (2010). Effect of balance training on postural instability in patients with idiopathic Parkinson's disease. Neurorehabilitation and neural repair, 24(9), 826-834. https://doi.org/10.1177\%2F1545968310376057.

Terrens, A. F., Soh, S. E., \& Morgan, P. (2020). The safety and feasibility of a Halliwick style of aquatic physiotherapy for falls and balance dysfunction in people with Parkinson's disease: a single blind pilot trial. PloS one, 15(7), e0236391. https://doi.org/10.1371/journal.pone.0236391.

Volpe, D., Giantin, M. G., Maestri, R., \& Frazzitta, G. (2014). Comparing the effects of hydrotherapy and land-based therapy on balance in patients with Parkinson's disease: a randomized controlled pilot study. Clinical rehabilitation, 28(12), 1210-1217.https://doi.org/10.1177/0269215514536060. 